\title{
La maintenance parodontale : aspects théoriques et pratiques
}

\section{Periodontal maintenance: theoretical and practical} aspects

\section{MOTS-CLEFS :}

- Parodontologie, maintenance, adhésion au traitement, thérapeutique parodontale de soutien.

\section{KEYWORDS:}

- Periodontology, maintenance, compliance, supportive periodontal therapy.
AOS 2014;267:12-19 DOI: $10.1051 / \mathrm{aos} / 2014103$ (C) EDP Sciences 2014

\section{Résumé}

Les maladies parodontales sont des infections polymicrobiennes dont le traitement se base essentiellement sur l'élimination des bactéries causales. Cette élimination ne peut être complète, d'où la notion du contrôle du facteur bactérien. En effet, la recolonisation bactérienne des sites traités se fait en quelques semaines et le retour des pathogènes parodontaux peut se produire en absence de contrôle de plaque continu par le praticien. Ainsi, une thérapeutique de maintenance parodontale se trouve justifiée pour soutenir le contrôle du facteur bactérien dans le temps et éviter la réactivation de la maladie parodontale. C'est une thérapeutique qui permet de préserver la denture et pérenniser les résultats du traitement parodontal actif. Son succès implique des facteurs liés au praticien et au patient. Le présent article se propose de traiter lintérêt de la maintenance parodontale, et de décrire les différents facteurs influençant son efficacité.

\author{
Abstract
}

Periodontal disease is a polymicrobial infection whose treatment is essentially based on the elimination of causal bacteria. This elimination can not be complete, which hence the notion of control of bacterial factor. In fact, the bacterial recolonization of treated sites takes place in a few weeks and the return of periodontal pathogens may occur in the absence of continuous plaque monitoring by the practitioner. Thus, a supportive periodontal therapy is necessary to maintain the control of the bacterial factor and prevent the reactivation of periodontal disease. This therapy preserves teeth and maintains the results of active periodontal treatment. Its success involves factors within the practitioner and patient. This article aims to show the interest of periodontal maintenance, and describe the various factors influencing its effectiveness.

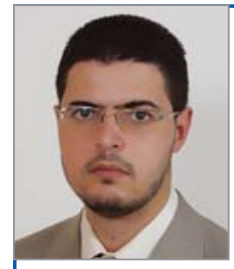

- A. HBIBI, Résident au Service de parodontologie. Faculté de médecine dentaire de Rabat. Université Mohammed V-Suissi. Maroc.

M. RHISSASSI, Chef du Département de Parodontologie. Faculté de médecine dentaire de Rabat. Université Mohammed V-Suissi. Maroc.

OK. ENNIBI, Professeur d'enseignement supérieur en parodontologie. Chef de service de parodontologie. Centre de consultation et traitement dentaire, Rabat, Maroc. 


\section{INTRODUCTION}

Les maladies parodontales sont des maladies inflammatoires multifactorielles, qui résultent d'une infection opportuniste par des bactéries couramment présentes dans la cavité buccale. Leur traitement se base sur la diminution de l'inflammation, et l'élimination des bactéries causales [1]. Cependant, cette désinfection ne peut pas être assurée pour de longues durées après traitement parodontal actif [2]. D'où l'intérêt de la maintenance parodontale dont l'objectif est de prévenir la récidive de la maladie. Cette récidive peut se produire dès qu'un site recommence à perdre de l'attache, après un changement local de la flore parodontale ou suite à un changement dans le comportement du patient ou après les deux à la fois. Il n'existe pas de critère simple pour prévenir la récidive, seul un programme rigoureux de maintenance de la santé parodontale permet de l'éviter. La maintenance parodontale, ou thérapeutique parodontale de soutien (TPS), est une étape clé de la thérapeutique parodontale. Il s'agit de l'ensemble de procédures réalisées à des intervalles déterminés afin d'assister le patient à maintenir sa santé orale [3].

Selon l'académie américaine de parodontologie les objectifs de la maintenance parodontale sont de trois ordres : minimiser les risques de récidive de la maladie parodontale chez des patients ayant suivi un traitement de gingivite ou de parodontite; réduire l'incidence de la perte des dents par la surveillance de la denture et de toute prothèse en bouche et accroitre la probabilité de traiter et de localiser, au moment opportun, d'autres maladies ou perturbations au sein de la cavité buccale [4]. La maintenance parodontale doit inclure l'actualisation du dossier médical du patient, l'évaluation de son état bucco-dentaire et de son hygiène orale et les facteurs de risque de la maladie parodontale, l'élimination de la plaque supra et sous-gingivale ainsi que du tartre et le retraitement des sites récidivants ou actifs [4]. Dans ce qui suit nous allons discuter l'intérêt de la maintenance parodontale dans le maintien de la santé parodontale ainsi que les facteurs pouvant influencer son efficacité.

\section{INTÉRÊT DE LA MAINTENANCE POUR LA SANTÉ PARODONTALE}

La récidive de la maladie parodontale survient souvent après un contrôle de plaque insuffisant de la part du patient ou du praticien. Cette situation qui implique la notion de réapparition de l'infection et de l'inflammation au niveau de certains sites préalablement traités s'accompagne d'une nouvelle perte d'attache. En effet, bien que le débridement des poches parodontales élimine les composants de la flore sous-gingivale associée aux parodontites, les pathogènes parodontaux peuvent recoloniser les sites traités en quelques jours ou quelque mois [5]. Cette recolonisation peut se produire en 9 à 11 semaines, mais peut varier sensiblement entre les patients [6-7] ; ce qui souligne l'intérêt d'une maintenance parodontale rigoureuse et individualisée. Différentes études s'accordent sur l'efficacité de la maintenance parodontale dans la stabilité des résultats du traitement parodontal (tableau 1). Ainsi, il a été montré que les patients placés sous un contrôle de plaque strict et permanent, conservent un niveau d'attache stable sur 6 ans, alors que les patients qui ne suivent pas un programme de contrôle de plaque présentent une récidive de la maladie parodontale [17]. Lindhe et Nyman [18] ont évalué les conditions parodontales après traitement actif, chez un groupe de patients présentant une parodontite sévère. Ils ont montré que, dans les conditions de parodontite sévère, la santé parodontale peut être maintenue plus de 14 ans si un programme de maintenance rigoureuse est établi. La moyenne des valeurs individuelles des profondeurs de sondage, des niveaux d'attache et les niveaux osseux n'ont pas varié significativement au cours des 14 années de suivi de l'étude, aussi bien chez les patients jeunes que chez les patients âgés. Ces résultats montrent l'intérêt de la maintenance dans la préservation de la santé parodontale par rapport à l'absence de traitement parodontal actif qui engendrerait une perte dentaire moyenne de 0,61\% par patient et par an [19]. De même, il a été rapporté qu'une maintenance régulière permet de maîtriser la progression de la maladie parodontale en assurant le contrôle des facteurs de risque [20-21].

\section{FACTEURS AFFECTANT L'EFFICACITÉ DE LA MAINTENANCE}

La stabilité du traitement parodontal est conditionnée par un suivi à long terme afin d'intercepter toute réactivation de la maladie. Ceci implique deux intervenants: le praticien et le patient.

\section{Facteur praticien}

La fréquence des rendez-vous, leur durée, la nature et la compétence du personnel impliqué dans la prise en charge sont autant d'éléments qui doivent être pris en considération en comparant les résultats des différentes études. Les premières études sur la maintenance parodontale ont été menées selon un programme 
$\checkmark$ Tableau 1 : Études sur l'intérêt de la maintenance parodontale

\begin{tabular}{|c|c|c|c|c|c|}
\hline Auteurs & $\begin{array}{l}\text { Nombre } \\
\text { de patients }\end{array}$ & $\begin{array}{l}\text { Type de } \\
\text { publication }\end{array}$ & $\begin{array}{l}\text { Paramètres } \\
\text { étudiés }\end{array}$ & $\begin{array}{l}\text { Durée } \\
\text { de l'étude } \\
\text { (moyenne) }\end{array}$ & $\begin{array}{l}\text { Résultats } \\
\text { de l'étude }\end{array}$ \\
\hline $\begin{array}{l}\text { Checchi et al. } \\
1994 \text { [8] }\end{array}$ & 192 patients & $\begin{array}{c}\text { Étude } \\
\text { rétrospective }\end{array}$ & I Perte dentaire & 6,7 ans & $\begin{array}{l}\text { Taux de perte dentaire faible chez } \\
\text { les patients ayant suivi un pro- } \\
\text { gramme de maintenance strict. }\end{array}$ \\
\hline $\begin{array}{l}\text { Kocher et al. } \\
2000 \text { [9] }\end{array}$ & $\begin{array}{l}\text { I } 14 \text { patients non traités } \\
\text { I } 26 \text { patients ayant abandonné la } \\
\text { thérapeutique de maintenance } \\
\text { après le traitement actif } \\
\text { I } 27 \text { patients ayant suivi une main- } \\
\text { tenance régulière }\end{array}$ & $\begin{array}{c}\text { Étude } \\
\text { rétrospective }\end{array}$ & $\begin{array}{l}\text { Changement radio- } \\
\text { graphique du niveau } \\
\text { d'os interdentaire } \\
\text { I Nombre de dents }\end{array}$ & 7 ans & $\begin{array}{l}\text { Le traitement parodontal actif } \\
\text { stoppe la perte osseuse interden- } \\
\text { taire et diminue le risque de perte } \\
\text { dentaire. La chirurgie parodon- } \\
\text { tale non suivie de maintenance } \\
\text { régulière ne permet pas prévenir } \\
\text { la progression de la destruction } \\
\text { parodontale. }\end{array}$ \\
\hline $\begin{array}{l}\text { König et al. } \\
2001 \text { [10] }\end{array}$ & $\begin{array}{l}\text { I } 142 \text { patients ayant eu un trai- } \\
\text { tement parodontal et un suivi } \\
\text { régulier en maintenance } \\
42 \text { patients ayant bénéficié d'un } \\
\text { traitement parodontal mais } \\
\text { dont le suivi en maintenance } \\
\text { était irrégulier } \\
44 \text { patients ayant abandonné le } \\
\text { traitement avant ou au cours du } \\
\text { traitement chirurgical }\end{array}$ & $\begin{array}{l}\text { Étude } \\
\text { rétrospective }\end{array}$ & $\begin{array}{l}\text { Indice de plaque } \\
\text { I Profondeur de poche } \\
\text { I Mobilité dentaire } \\
\text { I Atteinte de furcation } \\
\text { I Nombre de dents }\end{array}$ & 10 ans & $\begin{array}{l}\text { Les patients ayant une main- } \\
\text { tenance irrégulière répondent } \\
\text { moins favorablement à la chirur- } \\
\text { gie et à la thérapeutique paro- } \\
\text { dontale de soutien. }\end{array}$ \\
\hline
\end{tabular}

\begin{tabular}{|c|c|c|c|c|c|}
\hline $\begin{array}{l}\text { Fardal et al. } \\
2004 \text { [11] }\end{array}$ & $\begin{array}{l}\text { I } 68 \text { femmes } \\
\text { I } 32 \text { hommes }\end{array}$ & $\begin{array}{l}\text { Étude } \\
\text { rétrospective }\end{array}$ & I Perte dentaire & $9-11$ ans & $\begin{array}{l}\text { La maintenance parodontale } \\
\text { suite au traitement actif est asso- } \\
\text { ciée à un taux faible de perte } \\
\text { dentaire. }\end{array}$ \\
\hline $\begin{array}{l}\text { Matuliene } \\
\text { et al. } \\
2010 \text { [12] }\end{array}$ & 160 patients & $\begin{array}{c}\text { Étude } \\
\text { prospective }\end{array}$ & $\begin{array}{l}\text { I Saignement } \\
\text { au sondage } \\
\text { I Perte dentaire } \\
\text { I Poche parodontale }\end{array}$ & 10 ans & $\begin{array}{l}\text { Les patients adhérents à un pro- } \\
\text { gramme de maintenance sont à } \\
\text { faible risque de récidive de mala- } \\
\text { die parodontale et de perte den- } \\
\text { taire par rapport aux patients à } \\
\text { maintenance irrégulière }\end{array}$ \\
\hline $\begin{array}{l}\text { Chambrone } \\
\text { et al. } 2006 \\
\text { [13] }\end{array}$ & 120 patients & $\begin{array}{l}\text { Étude } \\
\text { rétrospective }\end{array}$ & I Perte dentaire & 10 ans & $\begin{array}{l}\text { Les patients traités et ayant suivi } \\
\text { un programme de maintenance } \\
\text { régulier ont présenté un faible } \\
\text { taux de perte dentaire. }\end{array}$ \\
\hline $\begin{array}{l}\text { Ng et al. } \\
2011 \text { [14] }\end{array}$ & $\begin{array}{l}\text { I } 273 \text { patients ayant suivi un pro- } \\
\text { gramme de maintenance } \\
39 \text { patients ayant arrêté la théra- } \\
\text { peutique de maintenance }\end{array}$ & $\begin{array}{l}\text { Étude } \\
\text { rétrospective }\end{array}$ & $\begin{array}{l}\text { Perte dentaire } \\
\text { par maladie } \\
\text { parodontale }\end{array}$ & 7 ans & $\begin{array}{l}\text { Les patients sous maintenance } \\
\text { parodontale perdent moins de } \\
\text { dents que les patients n'ayant } \\
\text { pas suivi une maintenance régu- } \\
\text { lière. }\end{array}$ \\
\hline $\begin{array}{l}\text { Fardal et al. } \\
2012[15]\end{array}$ & $\begin{array}{l}128 \text { femmes } \\
191 \text { hommes }\end{array}$ & $\begin{array}{l}\text { Étude } \\
\text { rétrospective }\end{array}$ & 1) Perte dentaire & 15 à 25 ans & $\begin{array}{l}\text { Les patients n'ayant pas suivi } \\
\text { une maintenance régulière ont } \\
\text { montré un taux élevé de perte } \\
\text { dentaire. }\end{array}$ \\
\hline $\begin{array}{l}\text { Costa et al. } \\
2012[16]\end{array}$ & $\begin{array}{l}\text { I } 288 \text { patients } \\
\text { In pratique privée et acadé- } \\
\text { mique publique }\end{array}$ & $\begin{array}{c}\text { Étude } \\
\text { prospective }\end{array}$ & $\begin{array}{l}\text { I Statut parodontal } \\
\text { I Progression de la } \\
\text { maladie parodontale } \\
\text { I Perte dentaire } \\
\text { I Influence sur les } \\
\text { facteurs de risque } \\
\text { prédictible }\end{array}$ & 1 an & $\begin{array}{l}\text { La thérapeutique de maintenance } \\
\text { minimise l'effet négatif des fac- } \\
\text { teurs de risque (tabac, diabète). }\end{array}$ \\
\hline
\end{tabular}


de rendez-vous très rigide. C'est ainsi que Nyman et al. [22] ont montré l'efficacité du contrôle de plaque professionnel toutes les deux semaines pendant deux ans pour maintenir les résultats de la chirurgie parodontale. Par ailleurs des études ultérieures ont montré qu'un intervalle de 3 à 4 mois était suffisant pour obtenir une bonne maintenance [23-25]. La plupart de ces études ont été menées dans des centres universitaires pour des périodes allant de 5 à 14 ans où les hygiénistes étaient largement impliqués dans la prise en charge avec des rendez-vous d'une durée d'une heure, soit 3 à 4 heures annuellement. En revanche, des études réalisées dans le secteur privé, ont montré une efficacité de la maintenance en accordant 30 minutes pour chaque rendez-vous avec entre 2 et 3 visites par an les 5 premières années et 2 visites pendant les 13 années suivantes. Cette durée représente la moitié de celle consacrée à la prise en charge des patients en milieu universitaire [26]. La comparaison des paramètres cliniques entre les études en milieu universitaire et privé montre une similitude des résultats mais la prise en charge semblerait moins chronophage en pratique privée. Par ailleurs, il est difficile de quantifier le facteur temps et coût des traitements de maintenance parodontale vu la diversité des variables impliquées, à savoir : la sévérité de la maladie, le nombre de dents restantes, la qualité et l'expérience clinique du personnel impliqué et les difficultés associées à chaque cas. Il est également intéressant d'étudier la différence des résultats cliniques entre la pratique générale et spécialisée. Pershaw et al. [27], ont montré que les résultats cliniques entre pratique générale et spécialisée au Royaume-Uni restent comparables à court terme pourvu que l'omnipraticien suive les instructions du praticien spécialiste avec un contrôle de plaque rigoureux. Toutefois, une méta-analyse plus récente [28] évaluant l'efficacité de la maintenance entre les deux types de pratique a conclu que la thérapeutique de maintenance parodontale chez un spécialiste a montré une meilleure stabilité des résultats du traitement parodontal et un meilleur taux de survie des dents.

\section{Facteur patient}

\section{Brossage dentaire}

La coopération du patient et l'application des instructions du parodontiste sont des éléments clés de la stabilité de l'état parodontal et de l'efficacité de la maintenance. Après les instructions d'hygiène nécessaires données par le praticien, le contrôle de plaque individuel fait partie de la responsabilité du patient envers sa maladie. Le patient doit être conscient de l'impact d'une hygiène bucco-dentaire correcte sur sa santé parodontale et générale. La fréquence de brossage, l'utilisation des moyens de brossage inter-proximal, le recours à des agents anti-plaque ou antibactériens, la dextérité manuelle du patient sont autant d'éléments qui influencent la qualité de l'hygiène buccodentaire. Plusieurs études cliniques et expérimentales ont confirmé que l'élimination de la plaque dentaire est essentielle pour la santé dentaire et parodontale [2932]. L'évolution des moyens de brossage dentaire et inter proximal a amélioré la qualité du contrôle de plaque individuel. Si aucune évidence scientifique en faveur d'un profil particulier de brosse à dents par rapport à un autre ne peut être réellement observé [31], la brosse à dents électrique avec un mouvement oscillatoire-rotatoire semble actuellement présenter une meilleure efficacité dans l'élimination de la plaque et la réduction de l'inflammation gingivale $[33,34]$. De plus, son utilisation serait d'un grand intérêt chez des patients à contrôle de plaque limité.

\section{Adhésion au traitement}

L'adhésion des patients au traitement est la condition sine qua non de la réussite de toute thérapeutique. Cette adhésion au traitement a été définie par Haynes [35] comme un état dans lequel le comportement du patient correspond aux instructions médicales données par le praticien pour améliorer sa santé. La première étude menée sur l'adhésion au programme de maintenance a été réalisée chez un groupe de patients traités en pratique privée aux Etats-Unis. Parmi 961 patients suivis pendant huit ans, seulement $16 \%$ ont adhéré complétement au programme de la maintenance, $34 \%$ ont été perdu de vue et le reste $(50 \%)$ consultait de façon irrégulière [36]. L'adhésion au traitement dans les centres de soins universitaires parait être similaire [37]. Plusieurs raisons peuvent justifier la non adhésion des patients à la thérapeutique de soutien : le manque de motivation du patient, la phobie des traitements dentaires, le coût du traitement, la situation socio-économique du patient, et la politique sanitaire du pays. Ces éléments peuvent moduler, entre autres, le comportement du patient envers sa santé orale. De plus, l'allongement du plan de traitement dans certains cas peut s'accompagner d'une lassitude progressive de la part du patient en termes de coopération. En effet, Ojima et al. (2005) [39], ont constaté que malgré la forte motivation des patients au début du traitement et leur inquiétude de perte des dents (plus de $90 \%$ ), seuls $52,7 \%$ des patients ont continué à consulter après 5 ans. La motivation du patient est un facteur indispensable mais insuffisant pour la pérennité de l'adhésion au traitement car elle ne peut pas garantir la continuité de sa coopération à long terme. Les conditions de vie des patients peuvent également influer sur leur coopération. En effet, une incidence élevée 
d'événements stressants dans la vie quotidienne [39] et la présence de conflit avec le supérieur hiérarchique [40] seraient des causes potentielles de non adhésion au traitement. D'autres facteurs psychologiques peuvent déterminer cette adhésion, à savoir : les croyances individuelles envers la santé, l'intelligence émotionnelle, et la personnalité [41]. Certaines attitudes envers la santé orale ont été également considérées comme étant des facteurs pronostiques significatifs d'une faible adhésion à long terme tels que : le manque de brossage de la gencive marginale, l'absence d'utilisation de brossettes ou fil interdentaires et la fréquence de consommation de boissons sucrées [38].

\section{Facteur financier}

Le rapport coût/efficacité de la maintenance a été mis au point, pour la première fois, en 2008, par Gaunt et al. [43]. Ces auteurs ont évalué, à travers une méta-analyse, l'efficacité de la maintenance prodiguée en pratique spécialisée et en omnipratique pour des patients atteints de parodontites chroniques. Les résultats montraient une meilleure stabilité parodontale et un meilleur taux de survie des dents en pratique spécialisée par rapport à l'omnipratique. Cependant, le coût de prise en charge revenait plus cher en pratique spécialisée. Ceci permet de conclure que le fait de conserver des dents avec un support parodontal réduit lors de la maintenance revient beaucoup moins cher que d'autres solutions alternatives (implant ou bridge) qui, de plus, présentent des risques de complications rendant leur entretien plus coûteux [44, 45].

\section{ANTIMICROBIENS AU COURS DE LA MAINTENANCE}

En parodontie, certains patients ont du mal à appliquer une hygiène buccodentaire parfaite, soit par présence de zones difficiles d'accès, soit par manque de dextérité. Pour compenser ce manque d'hygiène, les chercheurs ont testé l'efficacité d'antimicrobiens à long terme lors de la maintenance parodontale. Les systèmes d'administration topique de ces molécules varient entre les dentifrices, les bains de bouches, des systèmes à libération prolongée et l'irrigation au niveau de poches parodontales.

\section{Antiseptiques \\ Triclosan}

Le triclosan, en usage buccal à long terme, a montré son efficacité sur le ralentissement de la progression de la maladie parodontale chez les patients adhérents aléatoirement au programme de maintenance [46].
Cependant, plus récemment, certaines réserves ont été émises au regard des effets indésirables que peut engendrer cette molécule. D'une part, il a été suggéré que l'utilisation du triclosan exposerait au risque de résistance bactérienne voire même une résistance croisée des bactéries vis-à-vis d'autres anti-microbiens $[47,48]$. D'autre part, la large exposition à ce produit favoriserait la survenue de certains troubles immunitaires et endocriniens $[49,50]$. En effet, la présence bio-accumulative du triclosan dans les tissus humains et dans l'environnement pourrait accroître les risques de troubles endocriniens, d'autant plus que sa présence dans le sang et les urines est actuellement confirmée [51, 52]. Les études précitées, montrant la toxicité du triclosan, ne différencient pas l'effet cumulé du triclosan, contenu dans les différents produits à diverses concentrations, de l'effet unique du triclosan à $0,3 \%$ contenu dans la pâte dentifrice. Cullinan et al. [53] ont étudié l'effet du dentifrice au triclosan à $0,3 \%$ sur la fonction thyroïdienne. Leur étude a suivi les patients utilisant ce dentifrice pendant cinq ans, leurs résultats n'ont montré aucun effet significativement délétère sur la fonction thyroïdienne mais n'écarte pas le risque de toxicité de cette molécule sur le plan immunitaire. Devant l'évidence scientifique actuelle sur les effets indésirables du triclosan, sa prescription pour usage dentaire quotidien et lors de la maintenance parodontale ne peut être justifiée, surtout en absence d'études écartant les risques de son utilisation buccale sur la santé générale à long terme.

\section{Chlorhexidine}

La chlorhexidine représente actuellement le chef de file des antiseptiques utilisés en parodontie, l'utilisation classique étant indiquée généralement après débridement mécanique. Toutefois, les parodontistes restent encore prudents quant à son utilisation au cours de la maintenance. Des études testant cette molécule, au cours de la maintenance, sous forme de système à libération prolongée à base d'éthyle cellulose délivré tous les trois mois chez 10 patients pendant deux ans, ont montré une réduction significative de profondeur de poche et un meilleur gain d'attache au niveau des sites test par rapport aux sites contrôle [54, 55]. Rodrigues et al. [56] ont également testé ce système sur une période de maintenance de six mois par rapport au débridement mécanique seul. Les deux protocoles ont donné des résultats similaires mais la réduction des poches profondes était meilleure lorsqu'elles étaient traitées par le système de libération prolongée. Des études supplémentaires sur ce système seraient nécessaires pour valider son efficacité au cours de la maintenance. Par ailleurs, la formule bain de bouche 
n'a pas bénéficié d'autant d'études sur son efficacité à long terme. Il est connu que l'usage prolongé de chlorhexidine en bain de bouche peut entraîner des colorations des dents et de langue, une altération du goût et des douleurs et des irritations des muqueuses avec les formules alcoolisées. Pour pallier à ces inconvénients, Quirynen et al. ont testé une nouvelle formule de chlorhexidine à $0,05 \%$ associé au chlorure de cétyle de pyridinium à $0,05 \%$, sans alcool, pendant six mois après débridement en bouche entière [57]. Cette formule a été comparée à la chlorhexidine à $0,2 \%$ avec alcool. Les deux formules ont montré une réduction d'indice de plaque et d'indice gingival ainsi qu'une réduction des bactéries anaérobies, mais avec une supériorité de la nouvelle formule dans la réduction des effets indésirables de la chlorhexidine notamment la coloration des dents et de la langue.

\section{Antibiotiques en maintenance}

Plusieurs études ont montré l'efficacité des antibiotiques en adjonction à la thérapeutique initiale comme supplément au débridement mécanique particulièrement dans les formes agressives et sévères $[58,61]$. Cependant, l'avantage de cette adjonction sur la stabilité de l'état parodontal, à long terme, au cours de la maintenance a été très peu étudié. Les premières études sur ce sujet ont évalué l'effet d'un système de libération prolongée de tétracycline au cours de la maintenance. Les résultats ont montré une réduction significative $\mathrm{du}$ taux de récurrence de la maladie parodontale sur une durée de 6 à 12 mois [62,63]. Mais, les résultats à long terme avec cette forme d'administration locale de tétracyclines restent inconnus. Une autre formule thérapeutique a été testée ces dernières années ; il s'agit de l'administration de dose sous-antimicrobienne de doxycycline $[64,65]$. Une méta-analyse récente [66] évaluant l'efficacité de cette thérapeutique à court terme (3 mois d'administration journalière et 9 mois de suivi post-thérapeutique, a montré son efficacité sur la réduction de la profondeur de poche et un gain d'attache significatif à court terme (1 année). Des études supplémentaires sont nécessaires pour valider les différents protocoles d'antibiotiques pour une maintenance parodontale à long terme. Elles doivent montrer leur innocuité en termes de développement de résistance bactérienne ou de résistance croisée à long terme avec le minimum d'effets indésirables.

\section{RECOMMANDATIONS CLINIQUES}

\section{Adhésion au traitement}

I Simplifier et personnaliser le discours en fonction de la personnalité du patient, ses besoins de traitement et son contexte socio-économique. L'indifférence du praticien envers ses malades n'aide pas à les faire adhérer à la maintenance.

I Expliquer au patient l'intérêt de la maintenance dans la longévité des dents, le rapport coût/efficacité par rapport aux traitements alternatifs, et son intérêt dans la prévention de certaines maladies générales associées à la maladie parodontale.

I Rappeler au patient son rendez-vous de maintenance. I Evaluer continuellement la motivation du patient et son adhésion au traitement, et relever tout comportement pouvant être un facteur pronostic pour l'inobservance dans le futur, afin de de l'intercepter au moment opportun.

I Adopter un discours positif avec le patient en l'encourageant et le félicitant pour chaque comportement positif envers sa santé orale.

I Personnaliser la prescription de l'ordonnance en fonction de l'âge du patient, de sa dextérité manuelle et de son contexte socio-économique. Il est souhaitable de privilégier autant que possible les formes galéniques et les moyens d'hygiène qui facilitent l'observance thérapeutique selon chaque cas.

\section{Antimicrobiens}

I Triclosan : devant le manque d'évidence scientifique sur l'innocuité et l'intérêt à long terme du triclosan à $0,3 \%$ contenu dans les pâtes dentifrices, sa prescription en maintenance parodontale doit être conçue avec réserve.

I Chlorhexidine : les systèmes à libération prolongée ont montré leur efficacité à des intervalles d'utilisation de 3 mois. Ces procédés ont été testés à court terme (6 mois à 2 ans). Cependant, l'effet de cette formule sur la flore sous-gingivale à long terme reste inconnu. Il en est de même pour la formule de chlorhexidine à $0,05 \%$ qui n'a été testée que pendant 6 mois.

$\checkmark$ Antibiotiques : les tétracyclines en usage local et systémique ont montré leur intérêt sur l'amélioration des conditions parodontales dans la première année de maintenance seulement. Ainsi, il apparait difficile de recommander leur utilisation vu le faible nombre d'études réalisées sur ce sujet.

\section{CONCLUSION}

La maintenance parodontale est justifiée par le risque de récidive des maladies parodontales et la difficulté de la motivation pour la majorité des patients. C'est une thérapeutique qui engage les compétences du parodontiste et l'adhésion du patient. Elle reste une étape thérapeutique essentielle pour la pérennité des résultats du traitement parodontal dans le temps. Elle 
permet de limiter les risques d'échec thérapeutique par le contrôle des facteurs de risque des maladies parodontales et doit de ce fait être individualisée. L'intérêt d'adjuvants antimicrobiens nécessite encore des études à haut niveau de preuve scientifique montrant leur réel intérêt dans la maintenance à long terme.

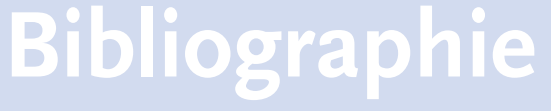

[1] Haffajee, A D. Systemic antibiotics: to use or not use in the treatment of periodontal infections. That is the question. J Clin Periodontol. 2006; 33, 359-361.

[2] Lang, N P, et al. Bleeding on probing. A predictor for the progression of periodontal desease. J Clin Periodontol. 1986,13:590,

[3] Académie Américaine de Parodontologie. Glossaire des termes parodontaux. 1992.

[4] Committee on Research, Science and Therapy of the American Academy of Periodontology. Supportive periodontal therapy. J Periodontol 1998: 69: 502-506.

[5] Slots J. The predominant cultivable microflora of advanced periodontitis. Scand J Dent Res 1977;85:114-121.

[6] Greenstein G. Periodontal response to mechanical non-surgical therapy: A review. J Peridontol 1992;63:118-130.

[7] Allen E, et al. Maintenance in periodontal therapy. Dent Update. 2008;35(3):150-2, 154-6.

[8] Checchi L, et al. Patient compliance with maintenance therapy in an Italian periodontal practice. J Clin Periodontol 1994;21:309-312.

[9] Kocher T, et al. Disease progression in periodontally treated and untreated patients a retrospective study. J Clin Periodontol 2000; 27: 866-872.

[10] König J, et al. Retrospective comparison of clinical variables between compliant and non-compliant patients. J Clin Periodontol 2001; 28 (3): 227-232.

[11] Fardal Ø, et al. Tooth loss during maintenance following periodontal treatment in a periodontal practice in Norway. J Clin Periodontol 2004; 31:550-555.

[12] Matuliene G, et al. Significance of Periodontal Risk Assessment on the recurrence of periodontitis and tooth loss. J Clin Periodontol 2010; 37: 191-199.

[13] Chambrone LA, Chambrone L. Tooth loss in well maintained patients with chronic periodontitis during long-term supportive therapy in Brazil. J Clin Periodontol 2006; 33: 759-764.
[14] Ng MC-H, et al. Tooth loss in compliant and noncompliant periodontally treated patients: 7 years after active periodontal therapy. J Clin Periodontol 2011; 38: 499-508.

[15] Fardal O, et al. Periodontal- and General Health in Long-Term Periodontal Maintenance Patients Treated in a Norwegian Private Practice. J Periodontol. $2012 ; 8$.

[16] Costa FO, et al. Prospective Study in Periodontal Maintenance Therapy: Comparative Analysis Between Academic and Private Practices. J Periodontol; 2012, 83(3):301-311.

[17] Axelsson P, Lindhe J. The significance of maintenance care in the treatment of periodontal disease. J Clin Periodontol 1981;8:281-294

[18] Lindhe J, Nyman S. Long term maintenance of patients treated for advanced periodontal disease. J Clin Periodontol 1983;10:148-156.

[19] Becker W, Berg L, Becker BE. Untreated periodontal disease: a longitudinal study. J Periodontol 1979;50:234-244.

[20] Darby M. Can we successfully maintain risk patients? Int J Dent Hygiene 2003, 1; 9-15.

[21] Costa OF, et al.Progression of periodontitis in a sample of regular and irregular compliers under maintenance therapy: a 3-year follow-up study. J Periodontol. 2011;82(9):1279-87.

[22] Nyman S, Rosling B, Lindhe J. Effect of professional toothcleaning on healing after periodontal surgery. J Clin Periodontol $1975 ; 2: 80-86$

[23] Knowles JW, et al.Results of periodontal treatment related to pocket depth and attachment level. Eight years. J Periodontol 1979;50:225-233.

[24] Lindhe J, Nyman S. Long-term maintenance of patients treated for advanced periodontal disease. J Clin Periodontol 1984;11:504-514.

[25] Pihlstrom BL, et al. Comparison of surgical and non-surgical treatment of periodontal disease. A review of current studies and additional results after 6.5 years.

J Clin Periodontol 1983;10:524-541.

[26] Tan AES. Longitudinal periodontal treatment outcomes in privatepractice. Ann R Australas Coll Dent Surg 2002;16:84-92.

[27] Preshaw PM, Heasman PA Periodontal maintenance in a specialist periodontal clinic and in general dental practice.

J Clin Periodontol 2005; 32: 280-286.
[28] Gaunt F, et al. The cost-effectiveness of supportive periodontal care for patients with chronic periodontitis. J Clin Periodontol 2008;35:67-82.

[29] Löe H, Theilade E, Jensen S. Experimental gingivitis in man. J Periodontol 1965: 36: 177-187.

[30] Löe H. Oral hygiene in the prevention of caries and periodontal disease. Int Dent J, 2000:50:129-139.

[31] Claydon NC. Current concepts in toothbrushing and interdental cleaning. Periodontol 2000. 2008;48:10-22.

[32] Van der Weijden F, Slot DE. Oral hygiene in the prevention of periodontal diseases: the evidence. Periodontology 2000, 2011;55:104-123.

[33] Dentino AR, et al. Six-month comparison of powered versus manual toothbrushing for safety and efficacy in the absence of professional instruction in mechanical plaque control. J Periodontol. 2002;73(7):770-8.

[34] Williams K, et al. Benefit of the power component of sonic and rotation-oscillation modes of action for plaque removal using power toothbrushes. Am J Dent. 2010 ;23(2):60-4.

[35] Haynes RB. A critical review of the "determinants" of patient compliance with therapeutic regimes. In: Sackett DL, Haynes RB, eds. Compliance with therapeutic regimens. Baltimore: John Hopkins University Press, 1976:2.

[36] Wilson TG Jr, et al. Compliance with maintenance therapy in a private periodontal practice. J Periodontol 1984;55:468-473.

[37] Aes T, Powell R, Seymour G. Patient attendance compliance in periodontal therapy. Aust Dent J 1992;37:467-471.

[38] Lorentz TCM, et al. Prospective study of complier individuals under periodontal maintenance therapy: analysis of clinical periodontal parameters, risk predictors and the progression of periodontitis. J Clin Periodontol 2009; 36: 58-67.

[39] Ojima M, Kanagawa H, Nishida N, Nagata H, Hanioka T, Shizukuishi S. Relationship between attitudes toward oral health at initial office visit and compliance with supportive periodontal treatment. J Clin Periodontol 2005; 32: 364-368.

[40] Becker BE, Karp C, Becker W, Berg L. Personality differences and stressful life events. Differences between treated periodontal patients with and without maintenance. J Clin Periodontol 1988;15:49-52. 
[41] Yeh H-C, Lai H. Association between patients' chief complaints and their compliance with periodontal therapy. J Clin Periodontol 2011; 38: 449-456.

[42] Umaki TM, et al. The Psychology of Patient Compliance: A Focused Review of the Literature. J Periodontol. 2012; 83(4):395-400.

[43] Gaunt F, et al. The cost-effectiveness of supportive periodontal care for patients with chronic periodontitis. J Clin Periodontol. 2008 ;35(8 Suppl):67-82.

[44] Pretzl B, et al. Effort and costs of tooth preservation in supportive periodontal treatment in a German population. J Clin Periodontol 2009; 36: 669-676.

[45] Pennington M, et al. The cost-effectiveness of supportive periodontal care: a global perspective. J Clin Periodontol 2011; 38:553-561.

[46] Cullinan MP, et al. The effect of a triclosan-containing dentifrice on the progression of periodontal disease in an adult population.

J Clin Periodontol 2003; 30; 414-419.

[47] Saleh S, Haddadin RN, Baillie S, Collier PJ. Triclosan - an update. Lett Appl Microbiol. $2011 ; 52(2): 87-95$.

[48] Dann AB, Hontela A. Triclosan: environmental exposure, toxicity and mechanisms of action. J Appl Toxicol. 2011 May;31(4):285-311.

[49] Dodson RE, Nishioka M, Standley LJ, Perovich LJ, Brody JG, Rudel RA. Endocrine disruptors and asthma-associated chemicals in consumer products. Environ Health Perspect. 2012 Jul;120(7):935-43.

[50] Clayton EM, Todd M, Dowd JB, Aiello AE. The impact of bisphenol A and triclosan on immune parameters in the U.S. population, NHANES 2003-2006. Environ Health Perspect. 2011;119(3):390-6.

[51] Hovander L, Malmberg T, Athanasiadou M, Athanassiadis I, Rahm S, Bergman A, Wehler; Identification of hydroxylated PCB metabolites and other phenolic halogenated pollutants in human blood plasma. Arch Environ Contam Toxicol. 2002;42(1):105-17.
[52] Pirard C, Sagot C, Deville M, Dubois $\mathrm{N}$, Charlier C. Urinary levels of bisphenol A, triclosan and 4-nonylphenol in a general Belgian population. Environ Int. 2012 Nov 1 ;48:78-83.

[53] Cullinan MP, Palmer JE, Carle AD, West MJ, Seymour GJ. Long term use of triclosan toothpaste and thyroid function. Sci Total Environ. 2012 Feb 1;416:75-9.

[54] Stabholz A, Soskolne WA, Friedman M, Sela MN. The use of sustained release delivery of chlorhexidine for the maintenance of periodontal pockets: 2-year clinical trial. J Periodontol. 1991;62(7):429-33.

[55] Soskolne WA, Proskin HM, Stabholz A. Probing depth changes following 2 years of periodontal maintenance therapy including adjunctive controlled release of chlorhexidine. J Periodontol. 2003;74(4):420-7.

[56] Rodrigues IF, Machion L, Casati MZ, Nociti FH Jr, de Toledo S, Sallum AW, Sallum EA. Clinical evaluation of the use of locally delivered chlorhexidine in periodontal maintenance therapy. J Periodontol. 2007;78(4):624-8.

[57] Quirynen M, Soers C, Desnyder M, Dekeyser C, Pauwels M, van Steenberghe D. A $0.05 \%$ cetyl pyridinium chloride $/ 0.05 \%$ chlorhexidine mouth rinse during maintenance phase after initial periodontal therapy. J Clin Periodontol. 2005 ;32(4):390-400.

[58] Yek EC, Cintan S, Topcuoglu N, Kulekci G, Issever H, Kantarci A. Efficacy of amoxicillin and metronidazole combination for the management of generalized aggressive periodontitis. J Periodontol. 2010 ;81(7):964-74.

[59] Mestnik MJ, Feres M, Figueiredo LC, Duarte PM, Lira EA, Faveri M.Short-term benefits of the adjunctive use of metronidazole plus amoxicillin in the microbial profile and in the clinical parameters of subjects with generalized aggressive periodontitis. J Clin Periodontol. 2010 ;37(4):353-65.
[60] Silva MP, Feres M, Sirotto TA, Soares GM, Mendes JA, Faveri M, Figueiredo LC. Clinical and microbiological benefits of metronidazole alone or with amoxicillin as adjuncts in the treatment of chronic periodontitis: a randomized placebo-controlled clinical trial. J Clin Periodontol. 2011;38(9):828-37.

[61] Cionca N, Giannopoulou C, Ugolotti G, Mombelli A.. Amoxicillin and metronidazole as an adjunct to full-mouth scaling and root planing of chronic periodontitis. J Periodontol. $2009 ; 80(3): 364-71$.

[62] Michalowicz BS, Pihlstrom BL, Drisko CL, Cobb CM, Killoy WJ, Caton JG, Lowenguth RA, Quinones C, Encarnacion M, Knowles M, Goodson JM. Evaluation of periodontal treatments using controlled release tetracycline fibers: maintenance response. J Periodontol 1995:66: 708-715.

[63] Cattabriga M, Pedrazzoli V, Cattabriga A, Pannutti E, Trapani M, Verrocchi GC. Tetracycline fiber used alone or with scaling and root planing in periodontal maintenance patients: clinical results. Quintessence Int 1996:27: 395-400.

[64] Emingil G, Atilla G, Sorsa T, Luoto H, Kirilmaz L, Baylas H. The effect of adjunctive low-dose doxycycline therapy on clinical parameters and gingival crevicular fluid matrix metalloproteinase-8 levels in chronic periodontitis. J Periodontol 2004;75:106-115.

[65] Emingil G, Gürkan A, Atilla G, Berdeli A, Cinarcik S. Adjunctive low-dose doxycycline therapy effect on clinical parameters and gingival crevicular fluid tissue plasminogen activator levels in chronic periodontitis. Inflamm Res 2006;55:550-558.

[66] Sgolastra F, Petrucci A, Gatto R, Giannoni M, Monaco A. Long-term efficacy of subantimicrobial-dose doxycycline as an adjunctive treatment to scaling and root planing: a systematic review and meta-analysis. J Periodontol. 2011;82(11):1570-81. 